\title{
Periodic track model for the prediction of railway induced vibration due to parametric excitation
}

\author{
M. Germonpré*, G. Degrande, G. Lombaert \\ KU Leuven, Department of Civil Engineering, Kasteelpark Arenberg 40, 3001 Leuven, Belgium
}

\begin{abstract}
Railway induced ground-borne vibration in urban areas has become a major environmental problem with large societal impact. Accurate computational models for the prediction of railway induced vibration generally require extensive modeling effort and computation time. To increase the computational efficiency, a large number of track models assume a simplified track structure in the longitudinal direction, therefore being unable to account for parametric excitation due to transition zones, hanging sleepers or spatially varying track or subgrade characteristics. This paper presents a track modeling approach based on a wave analysis technique for multi-coupled periodic structures that allows to efficiently model a track with varying characteristics in the longitudinal direction and, therefore, to predict railway induced vibration due to parametric excitation. The track is modeled by a finite number of track sections with different properties, while each section consists of periodic cells with the same properties. A case study is presented investigating a track with a transition zone between ballasted and slab track. The train-track interaction forces are computed, as well as the vibration of the sleepers, the slab and in the free field. The influence of the train direction and train speed is evaluated.
\end{abstract}

Keywords: Track modeling, parametric excitation, train-track interaction, railway induced vibration, transition zone

\section{Introduction}

To study railway induced ground-borne vibration, numerical track models are of great importance. The largest flexibility in modeling complex track and soil geometries is obtained by a fully three-dimensional (3D) finite element (FE) model $[1,2]$. The infinite dimensions of the problem domain must be taken into account to avoid spurious reflections at the boundaries of the finite volume of soil. This is often solved by means of a coupled finite element-boundary element (FE-BE) approach $[3,4]$. The main disadvantage of $3 \mathrm{D}$ models is that they require extensive modeling effort and computation time. A large number of authors

\footnotetext{
*Corresponding author. Tel.: +32 (0) 16320421.

Email address: matthias.germonpre@kuleuven.be (M. Germonpré) Postprint submitted to Transportation Geotechnics

Published version: M. Germonpré, G. Degrande and G. Lombaert. Periodic track model for the prediction of railway induced vibration due to parametric excitation. Transportation Geotechnics, 17(A): 98-108, 2018. https://doi.org/10.1016/j.trgeo.2018.09.015
} 
have therefore exploited the regularity of the track geometry to develop simplified track models that are computationally more efficient.

In a 2.5D FE-BE approach, the track and soil are assumed to be invariant in the longitudinal direction. A Fourier transformation of the longitudinal coordinate facilitates an efficient solution in the frequencywavenumber domain [5]. Only a 2D cross section of the track has to be meshed, allowing for a detailed representation of the cross sectional geometry. Due to the assumption of an invariant geometry, these models cannot take into account parametric excitation as arising, for example, from the periodic rail support in ballasted tracks.

Alternatively, the track can be assumed to be periodic to take into account the periodicity of the track structure. A Floquet transformation $[6,7,8]$ enables a solution based on the discretization of a single 3D periodic cell. This allows to account for parametric excitation due to the periodic rail support by sleepers, but not to model other sources of track stiffness variation, such as hanging sleepers, transition zones or spatial variations of the subgrade stiffness. Arlaud et al. $[9,10]$ developed a similar model, where the track is divided into periodic cells of which the modes are computed. This approach allows to significantly reduce the number of degrees of freedom compared to a 3D finite element model. Other sources of track stiffness variation can be studied as well, but the computational efficiency reduces significantly in this case. The prediction of the response of the track or receivers close to the track is very efficient, but the model is less suited for response predictions at larger distances from the track, since the soil domain is modeled with fixed boundaries and therefore no absorption of waves is accounted.

For the study of singular defects such as rail joints, switches and crossings, Kouroussis et al. [11] propose a two-step approach in which the train-track interaction is simulated using a flexible track model with a coupled lumped mass model to represent the soil foundation. The forces acting on the foundation are calculated and used as input for the second submodel of the soil which is described using a 3D finite/infinite element approach. The method is computationally efficient, but it does not allow to accurately account for the detailed geometry of a transition zone. Also, using two soil models can lead to additional modelling errors.

This paper presents a track modeling approach based on a wave analysis technique for multi-coupled periodic structures [12] that allows to efficiently model a track with varying characteristics in the longitudinal direction and, therefore, to predict railway induced vibration due to parametric excitation. The advantage of this technique is that it allows to model any kind of track stiffness variation, such as transition zones, tracks with hanging sleepers or with spatial variations of the ballast and subgrade stiffness, while it is computationally much more efficient than a 3D model. This track model is used in a case study of a transition zone between a slab track and a ballasted track on a high speed railway line in Chauconin, France, as also studied by Arlaud [9]. The passage of a high speed train on the transition zone is simulated. The train-track interaction forces are computed, as well as the vibration of the sleepers, the slab and the free field. The influence of the 
train direction and train speed is evaluated.

\section{Track model based on wave analysis technique}

\subsection{Description of the model}

A track modeling approach is presented based on the wave analysis technique for multi-coupled periodic structures [12]. The track is modeled by a finite number of track sections with different properties, while each section consists of periodic cells with the same properties. With this approach, the effect of parametric excitation due to variations in track geometry and track stiffness in the longitudinal direction can be taken into account while the total number of degrees of freedom never exceeds the number of degrees of freedom of one cell. Therefore, this approach significantly reduces the computation time and memory requirements compared to a fully 3D FE-BE model. This modeling approach was introduced and verified by means of a comparison with a periodic FE-BE model using the Floquet transform in [13]. A periodic ballasted track was modeled with both techniques and the vertical rail receptance as well as the track-free field mobility were compared. The results of both models correspond very well. The computation times of both modeling approaches were also compared and it was found that the track model based on the wave analysis technique on average needs ten times less computation time than the periodic FE-BE model.

Consider a track divided into $M$ parts with different properties (figure 1), with a central region of $M-2$ parts and semi-infinite series of cells extending infinitely to the left (part 1) and to the right (part $M$ ). Each part $j$ of the track consists of $N_{j}$ periodic cells with length $L_{j}$. These parts are separated by $M-1$ interfaces.

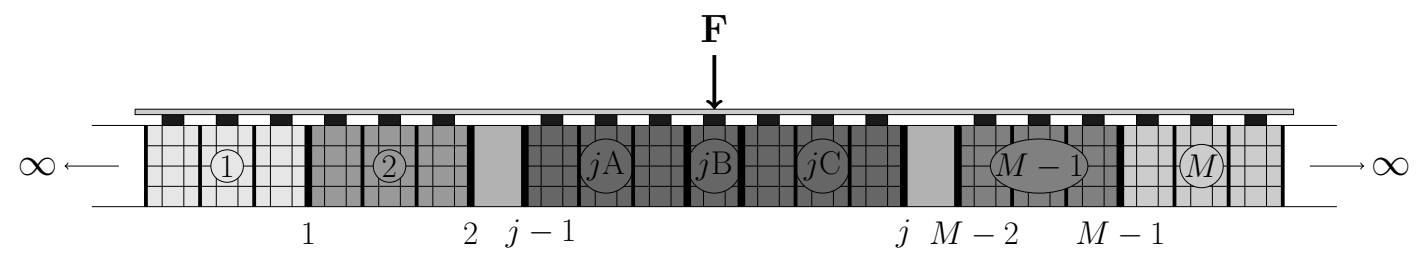

Figure 1: Track consisting of $M$ types of track structure, separated by $M-1$ interfaces, and partition of part $j$.

When a force $\mathbf{F}$ is applied to one of the cells of part $j$, this part of the track is split into three sub-parts (figure 1): the cell on which the force is applied (part $j \mathrm{~B}$ ), the cells of part $j$ left of this cell (part $j \mathrm{~A}$ ) and the cells of part $j$ right of this cell (part $j \mathrm{C}$ ). Part $j \mathrm{~A}$ or $j \mathrm{C}$ is not present when the force is applied on the first or last cell of part $j$, respectively. If the force is applied on a cell of part 1 , this part is split into the semi-infinite part 1A and the finite parts 1B and 1C. Similarly, if a force is applied on a cell of part $M$, this part is split into the finite parts $M \mathrm{~A}$ and $M \mathrm{~B}$ and the semi-infinite part $M \mathrm{C}$. 


\subsection{Computation of the free wave modes}

The displacement and force vectors $\mathbf{u}$ and $\mathbf{F}$ of each cell are partitioned into three parts: the nodes on the left boundary, the interior nodes and the nodes on the right boundary, referred to by the indices $\mathrm{L}$, I and $\mathrm{R}$, respectively. In free vibration, the displacements at the left-hand side of cells $k$ and $k+1$ (figure 2) are related by the propagation constant $\mu$ of the free wave:

$$
\mathbf{u}_{\mathrm{L} k+1}=e^{-\mu} \mathbf{u}_{\mathrm{L} k} .
$$

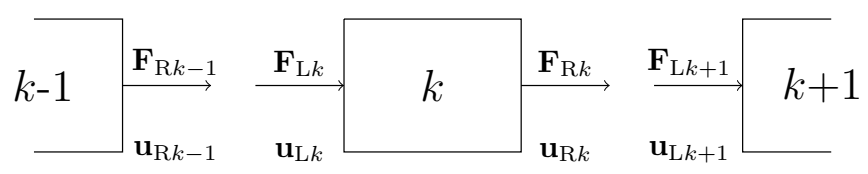

Figure 2: Displacements and forces on cell $k$.

Analogously, the forces at the left-hand side of cells $k$ and $k+1$ are related as:

$$
\mathbf{F}_{\mathrm{L} k+1}=e^{-\mu} \mathbf{F}_{\mathrm{L} k} .
$$

The computation of the free waves at each frequency is discussed in detail in [13] and is briefly summarized in this paper. The displacements and forces at the left- and right-hand side of cell $k$ are related as follows:

$$
\left[\begin{array}{cc}
\tilde{\mathbf{S}}_{\mathrm{LL}} & \tilde{\mathbf{S}}_{\mathrm{LR}} \\
\tilde{\mathbf{S}}_{\mathrm{RL}} & \tilde{\mathbf{S}}_{\mathrm{RR}}
\end{array}\right]\left\{\begin{array}{l}
\mathbf{u}_{\mathrm{L} k} \\
\mathbf{u}_{\mathrm{R} k}
\end{array}\right\}=\left\{\begin{array}{l}
\mathbf{F}_{\mathrm{L} k} \\
\mathbf{F}_{\mathrm{R} k}
\end{array}\right\},
$$

with $\tilde{\mathbf{S}}$ the dynamic stiffness matrix of the cell after condensation of the interior degrees of freedom. Combining equations (1), (2) and (3) and enforcing continuity of displacements and equilibrium of forces at the interface between two cells results in the following standard eigenvalue problem for each cell type, from which the free wave eigenvectors and propagation constants are found:

$$
\left[\begin{array}{cc}
\mathbf{0} & \mathbf{I} \\
-\tilde{\mathbf{S}}_{\mathrm{LR}}^{-1} \tilde{\mathbf{S}}_{\mathrm{RL}} & -\tilde{\mathbf{S}}_{\mathrm{LR}}^{-1}\left(\tilde{\mathbf{S}}_{\mathrm{LL}}+\tilde{\mathbf{S}}_{\mathrm{RR}}\right)
\end{array}\right]\left\{\begin{array}{l}
\mathbf{u}_{\mathrm{L} k} \\
\mathbf{u}_{\mathrm{R} k}
\end{array}\right\}=e^{-\mu}\left\{\begin{array}{c}
\mathbf{u}_{\mathrm{L} k} \\
\mathbf{u}_{\mathrm{R} k}
\end{array}\right\} .
$$

If the condition number of the coefficient matrix in equation (4) is too low to obtain accurate results, the wave modes can be found from the following equation, after appropriately scaling the unit matrices by a scalar $\sigma[14]$ :

$$
\left[\begin{array}{cc}
\tilde{\mathbf{S}}_{\mathrm{RL}} & \tilde{\mathbf{S}}_{\mathrm{RR}} \\
\mathbf{0} & \sigma \mathbf{I}
\end{array}\right]\left\{\begin{array}{l}
\mathbf{u}_{\mathrm{L} k} \\
\mathbf{u}_{\mathrm{R} k}
\end{array}\right\}=e^{-\mu}\left[\begin{array}{cc}
-\tilde{\mathbf{S}}_{\mathrm{LL}} & -\tilde{\mathbf{S}}_{\mathrm{LR}} \\
\sigma \mathbf{I} & \mathbf{0}
\end{array}\right]\left\{\begin{array}{l}
\mathbf{u}_{\mathrm{L} k} \\
\mathbf{u}_{\mathrm{R} k}
\end{array}\right\} .
$$

Alternatively, the following polynomial eigenvalue problem can be solved to obtain the free wave eigenvectors and propagation constants:

$$
\left[\tilde{\mathbf{S}}_{\mathrm{RL}}+e^{-\mu}\left(\tilde{\mathbf{S}}_{\mathrm{LL}}+\tilde{\mathbf{S}}_{\mathrm{RR}}\right)+e^{-2 \mu} \tilde{\mathbf{S}}_{\mathrm{LR}}\right] \mathbf{u}_{\mathrm{L} k}=\mathbf{0}
$$


At the interface between two cells, $n_{\mathrm{c}}$ degrees of freedom exist. Therefore, $n_{\mathrm{c}}$ free waves can propagate in both directions, each wave characterized by its wave eigenvector and complex propagation constant $\mu$, found from the eigenvalues $e^{-\mu}$ of equation (4), (5) or (6). The real part of a propagation constant determines the attenuation of the wave between two cells, while the imaginary part represents the change in phase of the wave per cell. The propagation constants are divided into positive-going waves, traveling from left to right in figure 1, and negative-going waves, traveling from right to left. Propagation constants with positive real part correspond to waves that decay when going from left to right, as can be observed from equation (1), and are therefore positive-going. Propagation constants with negative real part correspond to waves that decay when going from right to left and are therefore negative-going.

Each propagation constant $\mu$ is associated with a normalized eigenvector $\psi$ and a normalized force vector $\boldsymbol{\rho}$. The force vectors are calculated as follows:

$$
\boldsymbol{\rho}=\left[\tilde{\mathbf{S}}_{\mathrm{LL}}+\tilde{\mathbf{S}}_{\mathrm{LR}} e^{-\mu}\right] \boldsymbol{\psi}
$$

for positive-going waves, and

$$
\boldsymbol{\rho}=\left[\tilde{\mathbf{S}}_{\mathrm{RR}}+\tilde{\mathbf{S}}_{\mathrm{RL}} e^{+\mu}\right] \boldsymbol{\psi}
$$

for negative-going waves. For each cell type $j$, the vectors $\boldsymbol{\psi}$ and $\boldsymbol{\rho}$ are collected in the matrices $\mathbf{\Psi}_{\mathrm{p}}^{j}$ and $\mathbf{R}_{\mathrm{p}}^{j}$ for positive-going waves and $\boldsymbol{\Psi}_{\mathrm{n}}^{j}$ and $\mathbf{R}_{\mathrm{n}}^{j}$ for negative-going waves. In free vibration, the displacements and forces at the interface between two cells of type $j$ are always linear combinations of the eigenmodes $\boldsymbol{\Psi}_{\mathrm{p}}^{j}$ and $\mathbf{\Psi}_{\mathrm{n}}^{j}$ and forces $\mathbf{R}_{\mathrm{p}}^{j}$ and $\mathbf{R}_{\mathrm{n}}^{j}$, respectively.

\subsection{Stiffness matrix at boundary of semi-infinite track section}

In the track structure shown in figure 1, part 1 consists of a semi-infinite series of cells extending infinitely to the left. All the waves in this part are negative-going. At the right-hand side of part 1 , the displacements $\mathbf{u}_{\mathrm{R}}^{1}$ are decomposed into linear combinations of the eigenmodes $\mathbf{\Psi}_{\mathrm{n}}^{1}$ :

$$
\mathbf{u}_{\mathrm{R}}^{1}=\Psi_{\mathrm{n}}^{1} \boldsymbol{\alpha}_{\mathrm{n}}^{1}
$$

with $\boldsymbol{\alpha}_{\mathrm{n}}^{1}$ the modal coordinates of the negative-going waves in part 1. Analogously, the forces $\mathbf{F}_{\mathrm{R}}^{1}$ are decomposed into linear combinations of the forces $\mathbf{R}_{\mathrm{n}}^{1}$ :

$$
\mathbf{F}_{\mathrm{R}}^{1}=\mathbf{R}_{\mathrm{n}}^{1} \boldsymbol{\alpha}_{\mathrm{n}}^{1}
$$

Eliminating $\boldsymbol{\alpha}_{\mathrm{n}}^{1}$ from equations (9) and (10) results in the following expression:

$$
\mathbf{F}_{\mathrm{R}}^{1}=\mathbf{R}_{\mathrm{n}}^{1}\left(\boldsymbol{\Psi}_{\mathrm{n}}^{1}\right)^{-1} \mathbf{u}_{\mathrm{R}}^{1}=\mathbf{S}_{\mathrm{R}}^{1} \mathbf{u}_{\mathrm{R}}^{1},
$$

with $\mathbf{S}_{\mathrm{R}}^{1}$ the dynamic stiffness matrix relating the forces and displacements at the right-hand side of the semi-infinite part 1. A similar procedure can be followed for the semi-infinite part $M$ of the track:

$$
\mathbf{F}_{\mathrm{L}}^{M}=\mathbf{R}_{\mathrm{p}}^{M}\left(\mathbf{\Psi}_{\mathrm{p}}^{M}\right)^{-1} \mathbf{u}_{\mathrm{L}}^{M}=\mathbf{S}_{\mathrm{L}}^{M} \mathbf{u}_{\mathrm{L}}^{M},
$$


with $\mathrm{S}_{\mathrm{L}}^{M}$ the dynamic stiffness matrix at the left-hand side of part $M$.

\subsection{Stiffness matrix of finite track section}

In parts $j$ from 2 to $M-1$, both positive- and negative-going waves propagate. The displacements between cells $k$ and $k+1$ of part $j$ are written as (figure 3 ):

$$
\mathbf{u}_{\mathrm{R} k}^{j}=\mathbf{u}_{\mathrm{L} k+1}^{j}=\boldsymbol{\Psi}_{\mathrm{n}}^{j} e^{+\left(N_{j}-k\right) \mu_{\mathrm{n}}^{j}} \boldsymbol{\alpha}_{\mathrm{n}}^{j}+\Psi_{\mathrm{p}}^{j} e^{-k \mu_{\mathrm{p}}^{j}} \boldsymbol{\alpha}_{\mathrm{p}}^{j} .
$$
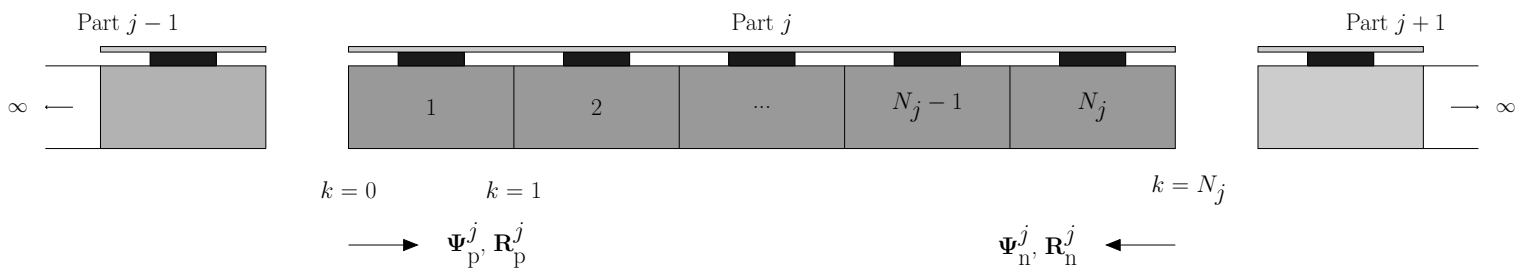

Figure 3: Wave modes in part $j$.

Similarly, the forces between cells $k$ and $k+1$ of part $j$ are written as:

$$
\mathbf{F}_{\mathrm{R} k}^{j}=-\mathbf{F}_{\mathrm{L} k+1}^{j}=\mathbf{R}_{\mathrm{n}}^{j} e^{+\left(N_{j}-k\right) \mu_{\mathrm{n}}^{j}} \boldsymbol{\alpha}_{\mathrm{n}}^{j}-\mathbf{R}_{\mathrm{p}}^{j} e^{-k \mu_{\mathrm{p}}^{j}} \boldsymbol{\alpha}_{\mathrm{p}}^{j} .
$$

The displacements and forces at the left-hand side of part $j$ are found for $k=0$ in equations (13) and (14), respectively. Similarly, the displacements and forces at the right-hand side of part $j$ are found for $k=N_{j}$. As a result, the displacements at the interfaces of part $j$ can be written as:

$$
\left\{\begin{array}{c}
\mathbf{u}_{\mathrm{L}}^{j} \\
\mathbf{u}_{\mathrm{R}}^{j}
\end{array}\right\}=\left\{\begin{array}{c}
\mathbf{u}_{\mathrm{L} 1}^{j} \\
\mathbf{u}_{\mathrm{R} N_{j}}^{j}
\end{array}\right\}=\left[\begin{array}{cc}
\boldsymbol{\Psi}_{\mathrm{p}}^{j} & \boldsymbol{\Psi}_{\mathrm{n}}^{j} e^{+N_{j} \mu_{\mathrm{n}}^{j}} \\
\boldsymbol{\Psi}_{\mathrm{p}}^{j} e^{-N_{j} \mu_{\mathrm{p}}^{j}} & \boldsymbol{\Psi}_{\mathrm{n}}^{j}
\end{array}\right]\left\{\begin{array}{l}
\boldsymbol{\alpha}_{\mathrm{p}}^{j} \\
\boldsymbol{\alpha}_{\mathrm{n}}^{j}
\end{array}\right\} .
$$

Analogously, the forces at the interfaces of part $j$ can be written as:

$$
\left\{\begin{array}{l}
\mathbf{F}_{\mathrm{L}}^{j} \\
\mathbf{F}_{\mathrm{R}}^{j}
\end{array}\right\}=\left\{\begin{array}{c}
\mathbf{F}_{\mathrm{L} 1}^{j} \\
\mathbf{F}_{\mathrm{R} N_{j}}^{j}
\end{array}\right\}=\left[\begin{array}{cc}
\mathbf{R}_{\mathrm{p}}^{j} & -\mathbf{R}_{\mathrm{n}}^{j} e^{+N_{j} \mu_{\mathrm{n}}^{j}} \\
-\mathbf{R}_{\mathrm{p}}^{j} e^{-N_{j} \mu_{\mathrm{p}}^{j}} & \mathbf{R}_{\mathrm{n}}^{j}
\end{array}\right]\left\{\begin{array}{l}
\boldsymbol{\alpha}_{\mathrm{p}}^{j} \\
\boldsymbol{\alpha}_{\mathrm{n}}^{j}
\end{array}\right\} .
$$

Eliminating the modal coordinates $\boldsymbol{\alpha}_{\mathrm{p}}^{j}$ and $\boldsymbol{\alpha}_{\mathrm{n}}^{j}$ from equations (15) and (16) yields an expression relating the forces and displacements at the left and right boundaries of part $j$ :

$$
\left\{\begin{array}{l}
\mathbf{F}_{\mathrm{L}}^{j} \\
\mathbf{F}_{\mathrm{R}}^{j}
\end{array}\right\}=\left[\begin{array}{cc}
\mathbf{R}_{\mathrm{p}}^{j} & -\mathbf{R}_{\mathrm{n}}^{j} e^{+N_{j} \mu_{\mathrm{n}}^{j}} \\
-\mathbf{R}_{\mathrm{p}}^{j} e^{-N_{j} \mu_{\mathrm{p}}^{j}} & \mathbf{R}_{\mathrm{n}}^{j}
\end{array}\right]\left[\begin{array}{cc}
\mathbf{\Psi}_{\mathrm{p}}^{j} & \mathbf{\Psi}_{\mathrm{n}}^{j} e^{+N_{j} \mu_{\mathrm{n}}^{j}} \\
\mathbf{\Psi}_{\mathrm{p}}^{j} e^{-N_{j} \mu_{\mathrm{p}}^{j}} & \mathbf{\Psi}_{\mathrm{n}}^{j}
\end{array}\right]^{-1}\left\{\begin{array}{c}
\mathbf{u}_{\mathrm{L}}^{j} \\
\mathbf{u}_{\mathrm{R}}^{j}
\end{array}\right\}=\left[\begin{array}{cc}
\tilde{\mathbf{S}}_{\mathrm{LL}}^{j} & \tilde{\mathbf{S}}_{\mathrm{LR}}^{j} \\
\tilde{\mathbf{S}}_{\mathrm{RL}}^{j} & \tilde{\mathbf{S}}_{\mathrm{RR}}^{j}
\end{array}\right]\left\{\begin{array}{c}
\mathbf{u}_{\mathrm{L}}^{j} \\
\mathbf{u}_{\mathrm{R}}^{j}
\end{array}\right\},
$$

with $\tilde{\mathbf{S}}^{j}$ the dynamic stiffness matrix of part $j$ after condensation of the degrees of freedom that do not belong to the left-hand or right-hand boundary of this part of the track. Using the properties of a block 
matrix, the submatrices of $\tilde{\mathbf{S}}^{j}$ are elaborated as:

$$
\begin{aligned}
& \tilde{\mathbf{S}}_{\mathrm{LL}}^{j}=+\left[\mathbf{R}_{\mathrm{p}}^{j}+\mathbf{R}_{\mathrm{n}}^{j} e^{+N_{j} \mu_{\mathrm{n}}^{j}}\left(\boldsymbol{\Psi}_{\mathrm{n}}^{j}\right)^{-1} \boldsymbol{\Psi}_{\mathrm{p}}^{j} e^{-N_{j} \mu_{\mathrm{p}}^{j}}\right]\left[\boldsymbol{\Psi}_{\mathrm{p}}^{j}-\boldsymbol{\Psi}_{\mathrm{n}}^{j} e^{+N_{j} \mu_{\mathrm{n}}^{j}}\left(\boldsymbol{\Psi}_{\mathrm{n}}^{j}\right)^{-1} \boldsymbol{\Psi}_{\mathrm{p}}^{j} e^{-N_{j} \mu_{\mathrm{p}}^{j}}\right]^{-1} \\
& \tilde{\mathbf{S}}_{\mathrm{LR}}^{j}=-\left[\mathbf{R}_{\mathrm{p}}^{j}\left(\boldsymbol{\Psi}_{\mathrm{p}}^{j}\right)^{-1} \boldsymbol{\Psi}_{\mathrm{n}}^{j} e^{+N_{j} \mu_{\mathrm{n}}^{j}}+\mathbf{R}_{\mathrm{n}}^{j} e^{+N_{j} \mu_{\mathrm{n}}^{j}}\right]\left[\boldsymbol{\Psi}_{\mathrm{n}}^{j}-\boldsymbol{\Psi}_{\mathrm{p}}^{j} e^{-N_{j} \mu_{\mathrm{p}}^{j}}\left(\boldsymbol{\Psi}_{\mathrm{p}}^{j}\right)^{-1} \boldsymbol{\Psi}_{\mathrm{n}}^{j} e^{+N_{j} \mu_{\mathrm{n}}^{j}}\right]^{-1} \\
& \tilde{\mathbf{S}}_{\mathrm{RL}}^{j}=-\left[\mathbf{R}_{\mathrm{n}}^{j}\left(\boldsymbol{\Psi}_{\mathrm{n}}^{j}\right)^{-1} \mathbf{\Psi}_{\mathrm{p}}^{j} e^{-N_{j} \mu_{\mathrm{p}}^{j}}+\mathbf{R}_{\mathrm{p}}^{j} e^{-N_{j} \mu_{\mathrm{p}}^{j}}\right]\left[\boldsymbol{\Psi}_{\mathrm{p}}^{j}-\boldsymbol{\Psi}_{\mathrm{n}}^{j} e^{+N_{j} \mu_{\mathrm{n}}^{j}}\left(\boldsymbol{\Psi}_{\mathrm{n}}^{j}\right)^{-1} \boldsymbol{\Psi}_{\mathrm{p}}^{j} e^{-N_{j} \mu_{\mathrm{p}}^{j}}\right]^{-1} \\
& \tilde{\mathbf{S}}_{\mathrm{RR}}^{j}=+\left[\mathbf{R}_{\mathrm{n}}^{j}+\mathbf{R}_{\mathrm{p}}^{j} e^{-N_{j} \mu_{\mathrm{p}}^{j}}\left(\boldsymbol{\Psi}_{\mathrm{p}}^{j}\right)^{-1} \boldsymbol{\Psi}_{\mathrm{n}}^{j} e^{+N_{j} \mu_{\mathrm{n}}^{j}}\right]\left[\boldsymbol{\Psi}_{\mathrm{n}}^{j}-\boldsymbol{\Psi}_{\mathrm{p}}^{j} e^{-N_{j} \mu_{\mathrm{p}}^{j}}\left(\boldsymbol{\Psi}_{\mathrm{p}}^{j}\right)^{-1} \boldsymbol{\Psi}_{\mathrm{n}}^{j} e^{+N_{j} \mu_{\mathrm{n}}^{j}}\right]^{-1} .
\end{aligned}
$$

The dynamic stiffness matrices $\tilde{\mathbf{S}}^{j \mathrm{~A}}$ and $\tilde{\mathbf{S}}^{j \mathrm{C}}$ are computed with equation (17), using $N_{j \mathrm{~A}}$ cells instead of $N_{j}$ cells in part $j \mathrm{~A}$ and $N_{j \mathrm{C}}$ cells in part $j \mathrm{C}$.

\subsection{Assembly of the system of equations}

Assembling the stiffness matrices of the different parts of the track and imposing continuity of displacements and equilibrium of forces at the interfaces, results in the following system of equations:

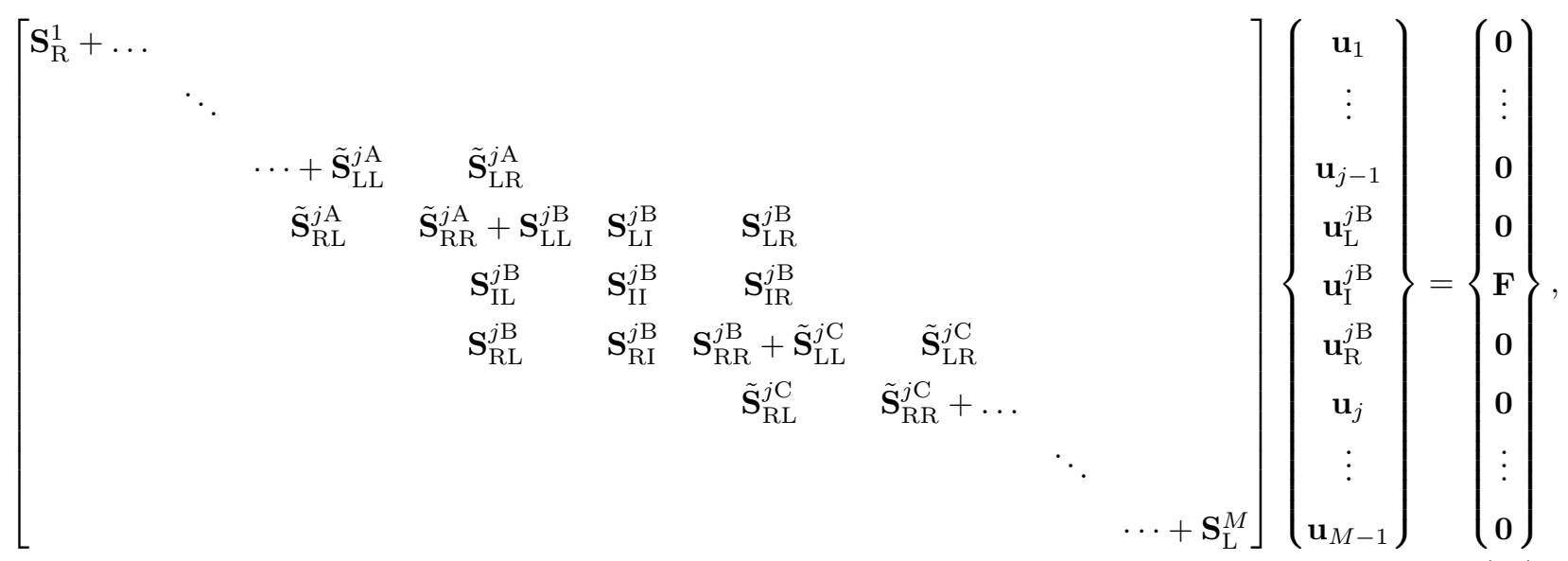

with $\mathbf{u}_{\mathrm{L}}^{j \mathrm{~B}}, \mathbf{u}_{\mathrm{I}}^{j \mathrm{~B}}$ and $\mathbf{u}_{\mathrm{R}}^{j \mathrm{~B}}$ the displacements at the left, interior and right of part $j \mathrm{~B}$ and $\mathbf{u}_{1}$ to $\mathbf{u}_{\mathrm{M}-1}$ the displacements of interfaces 1 to $M-1$. In the particular case where the force is applied to the first cell of part $j$, part $j \mathrm{~A}$ does not exist and $\mathbf{u}_{j-1}=\mathbf{u}_{\mathrm{L}}^{j \mathrm{~B}}$. The matrix $\tilde{\mathbf{S}}^{j \mathrm{~A}}$ is then replaced by $\tilde{\mathbf{S}}^{j-1}$. Similarly, if the force is applied on the last cell of part $j$, part $j \mathrm{C}$ does not exist, $\mathbf{u}_{j}=\mathbf{u}_{\mathrm{R}}^{j \mathrm{~B}}$ and the matrix $\tilde{\mathbf{S}}^{j \mathrm{C}}$ is replaced by $\tilde{\mathbf{S}}^{j+1}$.

\subsection{Sequence of equations for moving force on track}

Consider a train traveling along the track, starting at cell $k$ in part $j$. Forces are subsequently applied to the cells from left to right and a strategy is followed that limits the number of computations as much as possible.

First, the dynamic stiffness matrix $\mathbf{S}$ of each cell type is computed, as well as the corresponding propagation constants $\boldsymbol{\mu}$, wave eigenvectors $\boldsymbol{\Psi}$ and force vectors $\mathbf{R}$. Next, the matrices $\mathbf{S}_{\mathrm{R}}^{1}, \mathbf{S}_{\mathrm{L}}^{M}$ and $\tilde{\mathbf{S}}^{2}$ to $\tilde{\mathbf{S}}^{M-1}$ are computed and stored. 
Gaussian elimination is performed at the left- and right-hand sides of part $j$ until interfaces $j-1$ and $j$ are reached, resulting in the following system of equations:

$$
\left[\begin{array}{ccccc}
\mathbf{S}_{\mathrm{R}}^{j-1}+\tilde{\mathbf{S}}_{\mathrm{LL}}^{j \mathrm{~A}} & \tilde{\mathbf{S}}_{\mathrm{LR}}^{j \mathrm{~A}} & & & \\
\tilde{\mathbf{S}}_{\mathrm{RL}}^{j \mathrm{~A}} & \tilde{\mathbf{S}}_{\mathrm{RR}}^{j \mathrm{~A}}+\mathbf{S}_{\mathrm{LL}}^{j \mathrm{~B}} & \mathbf{S}_{\mathrm{LI}}^{j \mathrm{~B}} & \mathbf{S}_{\mathrm{LR}}^{j \mathrm{~B}} & \\
& \mathbf{S}_{\mathrm{IL}}^{j \mathrm{~B}} & \mathbf{S}_{\mathrm{II}}^{j \mathrm{~B}} & \mathbf{S}_{\mathrm{IR}}^{j \mathrm{~B}} & \\
& \mathbf{S}_{\mathrm{RL}}^{j \mathrm{~B}} & \mathbf{S}_{\mathrm{RI}}^{j \mathrm{~B}} & \mathbf{S}_{\mathrm{RR}}^{j \mathrm{~B}}+\tilde{\mathbf{S}}_{\mathrm{LL}}^{j \mathrm{C}} & \tilde{\mathbf{S}}_{\mathrm{LR}}^{j \mathrm{C}} \\
& & & \tilde{\mathbf{S}}_{\mathrm{RL}}^{j \mathrm{C}} & \tilde{\mathbf{S}}_{\mathrm{RR}}^{j \mathrm{C}}+\mathbf{S}_{\mathrm{L}}^{j+1}
\end{array}\right]\left\{\begin{array}{c}
\mathbf{u}_{j-1} \\
\mathbf{u}_{\mathrm{L}}^{j \mathrm{~B}} \\
\mathbf{u}_{\mathrm{I}}^{j \mathrm{~B}} \\
\mathbf{u}_{\mathrm{R}}^{j \mathrm{~B}} \\
\mathbf{u}_{j}
\end{array}\right\}=\left\{\begin{array}{c}
\mathbf{0} \\
\mathbf{0} \\
\mathbf{F} \\
\mathbf{0} \\
\mathbf{0}
\end{array}\right\},
$$

with $\mathbf{S}_{\mathrm{R}}^{j-1}$ the stiffness matrix relating the forces and displacements at the right-hand side of part $j-1$, computed by means of the following recursive relation:

$$
\mathbf{S}_{\mathrm{R}}^{l}=\tilde{\mathbf{S}}_{\mathrm{RR}}^{l}-\tilde{\mathbf{S}}_{\mathrm{RL}}^{l}\left[\mathbf{S}_{\mathrm{R}}^{l-1}+\tilde{\mathbf{S}}_{\mathrm{LL}}^{l}\right]^{-1} \tilde{\mathbf{S}}_{\mathrm{LR}}^{l}
$$

with $l=2$ to $j-1$. Similarly, $\mathbf{S}_{\mathrm{L}}^{j+1}$ is the stiffness matrix relating the forces and displacements at the left-hand side of part $j+1$, computed by means of the following recursive relation:

$$
\mathbf{S}_{\mathrm{L}}^{l}=\tilde{\mathbf{S}}_{\mathrm{LL}}^{l}-\tilde{\mathbf{S}}_{\mathrm{LR}}^{l}\left[\mathbf{S}_{\mathrm{L}}^{l+1}+\tilde{\mathbf{S}}_{\mathrm{RR}}^{l}\right]^{-1} \tilde{\mathbf{S}}_{\mathrm{RL}}^{l}
$$

with $l=M-1$ to $j+1$. The matrices $\mathbf{S}_{\mathrm{R}}^{1}$ to $\mathbf{S}_{\mathrm{R}}^{j-1}$ and $\mathbf{S}_{\mathrm{L}}^{M}$ to $\mathbf{S}_{\mathrm{L}}^{j+1}$ are stored, because they will be used to compute displacements outside part $j$, and to perform Gaussian elimination when the exterior force is applied on other parts than part $j$.

When forces are applied on different cells of part $j$, only the stiffness matrices $\tilde{\mathbf{S}}^{j \mathrm{~A}}$ and $\tilde{\mathbf{S}}^{j \mathrm{C}}$ change and the following steps are repeated for every cell $k$. The matrices $\tilde{\mathbf{S}}^{j \mathrm{~A}}$ and $\tilde{\mathbf{S}}^{j \mathrm{C}}$ are computed with equation (17) using $N_{j \mathrm{~A}}=k-1$ and $N_{j \mathrm{C}}=N_{j}-k$. Gaussian elimination is then performed until the left and right boundary of part $j \mathrm{~B}$ are reached:

$$
\left[\begin{array}{ccc}
\mathbf{S}_{\mathrm{R}}^{j \mathrm{~A}}+\mathbf{S}_{\mathrm{LL}}^{j \mathrm{~B}} & \mathbf{S}_{\mathrm{LI}}^{j \mathrm{~B}} & \mathbf{S}_{\mathrm{LR}}^{j \mathrm{~B}} \\
\mathbf{S}_{\mathrm{IL}}^{j \mathrm{~B}} & \mathbf{S}_{\mathrm{II}}^{j \mathrm{~B}} & \mathbf{S}_{\mathrm{IR}}^{j \mathrm{~B}} \\
\mathbf{S}_{\mathrm{RL}}^{j \mathrm{~B}} & \mathbf{S}_{\mathrm{RI}}^{j \mathrm{~B}} & \mathbf{S}_{\mathrm{RR}}^{j \mathrm{~B}}+\mathbf{S}_{\mathrm{L}}^{j \mathrm{C}}
\end{array}\right]\left\{\begin{array}{c}
\mathbf{u}_{\mathrm{L}}^{j \mathrm{~B}} \\
\mathbf{u}_{\mathrm{I}}^{j \mathrm{~B}} \\
\mathbf{u}_{\mathrm{R}}^{j \mathrm{~B}}
\end{array}\right\}=\left\{\begin{array}{c}
\mathbf{0} \\
\mathbf{F} \\
\mathbf{0}
\end{array}\right\}
$$

The stiffness matrix $\mathbf{S}_{\mathrm{R}}^{j \mathrm{~A}}$ relating the forces and displacements at the right-hand side of part $j \mathrm{~A}$ is computed as:

$$
\mathbf{S}_{\mathrm{R}}^{j \mathrm{~A}}=\tilde{\mathbf{S}}_{\mathrm{RR}}^{j \mathrm{~A}}-\tilde{\mathbf{S}}_{\mathrm{RL}}^{j \mathrm{~A}}\left[\mathbf{S}_{\mathrm{R}}^{j-1}+\tilde{\mathbf{S}}_{\mathrm{LL}}^{j \mathrm{~A}}\right]^{-1} \tilde{\mathbf{S}}_{\mathrm{LR}}^{j \mathrm{~A}}
$$

while the stiffness matrix $\mathbf{S}_{\mathrm{L}}^{j \mathrm{C}}$ relating the forces and displacements at the left-hand side of part $j \mathrm{C}$ is computed as:

$$
\mathbf{S}_{\mathrm{L}}^{j \mathrm{C}}=\tilde{\mathbf{S}}_{\mathrm{LL}}^{j \mathrm{C}}-\tilde{\mathbf{S}}_{\mathrm{LR}}^{j \mathrm{C}}\left[\mathbf{S}_{\mathrm{L}}^{j+1}+\tilde{\mathbf{S}}_{\mathrm{RR}}^{j \mathrm{C}}\right]^{-1} \tilde{\mathbf{S}}_{\mathrm{RL}}^{j \mathrm{C}}
$$

Equation (26) yields the displacements of part $j$ B. The displacements at interface $j-1$ are found from the first row of equation (3), taking into account that $\mathbf{F}_{\mathrm{L}}^{j \mathrm{~A}}=-\mathbf{S}_{\mathrm{R}}^{j-1} \mathbf{u}_{j-1}$ :

$$
\mathbf{u}_{j-1}=-\left[\tilde{\mathbf{S}}_{\mathrm{LL}}^{j \mathrm{~A}}+\mathbf{S}_{\mathrm{R}}^{j-1}\right]^{-1} \tilde{\mathbf{S}}_{\mathrm{LR}}^{j \mathrm{~A}} \mathbf{u}_{\mathrm{L}}^{j \mathrm{~B}}
$$


Analogously, the displacements at interface $j$ are found from the second row of equation (3), taking into account that $\mathbf{F}_{\mathrm{R}}^{j \mathrm{C}}=-\mathrm{S}_{\mathrm{L}}^{j+1} \mathbf{u}_{j}$ :

$$
\mathbf{u}_{j}=-\left[\tilde{\mathbf{S}}_{\mathrm{RR}}^{j \mathrm{C}}+\mathbf{S}_{\mathrm{L}}^{j+1}\right]^{-1} \tilde{\mathbf{S}}_{\mathrm{RL}}^{j \mathrm{C}} \mathbf{u}_{\mathrm{R}}^{j \mathrm{~B}} .
$$

The displacements at the other interfaces between the parts of the track are found from similar expressions:

$$
\mathbf{u}_{l-1}=-\left[\tilde{\mathbf{S}}_{\mathrm{LL}}^{l}+\mathbf{S}_{\mathrm{R}}^{l-1}\right]^{-1} \tilde{\mathbf{S}}_{\mathrm{LR}}^{l} \mathbf{u}_{l},
$$

for $l=j-1$ to 2 , and

$$
\mathbf{u}_{l}=-\left[\tilde{\mathbf{S}}_{\mathrm{RR}}^{l}+\mathbf{S}_{\mathrm{L}}^{l+1}\right]^{-1} \tilde{\mathbf{S}}_{\mathrm{RL}}^{l} \mathbf{u}_{l-1} .
$$

for $l=j+1$ to $M-1$.

To compute the displacements inside parts 1 to $j-1, j \mathrm{~A}, j \mathrm{C}$ and $j+1$ to $M$, the modal coordinates are calculated from the displacements at the interfaces using equation (9) or (15). The displacements at the interfaces between cells inside each part are found from these modal coordinates using equation (13). The interior displacements of each cell $k$ inside part $l$ are computed from the displacements at its left and right boundary:

$$
\mathbf{u}_{\mathrm{I} k}^{l}=-\left[\mathbf{S}_{\mathrm{II}}^{l}\right]^{-1}\left[\mathbf{S}_{\mathrm{IL}}^{l} \mathbf{u}_{\mathrm{L} k}^{l}+\mathbf{S}_{\mathrm{IR}}^{l} \mathbf{u}_{\mathrm{R} k}^{l}\right] .
$$

When forces are subsequently applied on the cells of part $j+1$, the same procedure is repeated.

\section{Case study: transition zone on a high speed line}

\subsection{Model of the transition zone and train}

The periodic track modeling approach is illustrated by a case study of a transition zone between ballasted track and slab track. It is investigated to what extent an appropriate design of the transition zone reduces the train-track interaction forces and sleeper vibration compared to a direct transition between the two track structures. Also, the effect of a speed reduction is studied.

Slab tracks are often used on bridges and in tunnels, due to their longer life span and reduced maintenance cost compared to ballasted tracks $[15,16]$. The difference in structural behavior between the two track systems makes the design of a transition zones challenging. This has triggered many numerical $[17,18]$ and experimental [19] studies. An extensive overview of transition zone designs and numerical track models is given in $[20]$.

The present study is based on an existing transition zone located in Chauconin on the high speed line LGV Est between Paris and Strasbourg. Arlaud [9] developed a model of this transition zone and validated it by means of measurements of the rail receptance and sleeper vibration. The track model implemented in the present publication is based on track geometry and material characteristics presented in [9]. This transition zone is particularly interesting since rapid track degradation was observed some time after opening the line 
for high speed traffic. Due to the appearance of hanging sleepers, the maximum train speed was reduced from $300 \mathrm{~km} / \mathrm{h}$ to $230 \mathrm{~km} / \mathrm{h}$.

The longitudinal geometry of the transition zone is shown in figure 4 and consists of four parts: the slab track, the ballasted track and a transition zone consisting of two track sections. The cross sectional geometry of the ballasted track and slab track are shown in figure 5 .

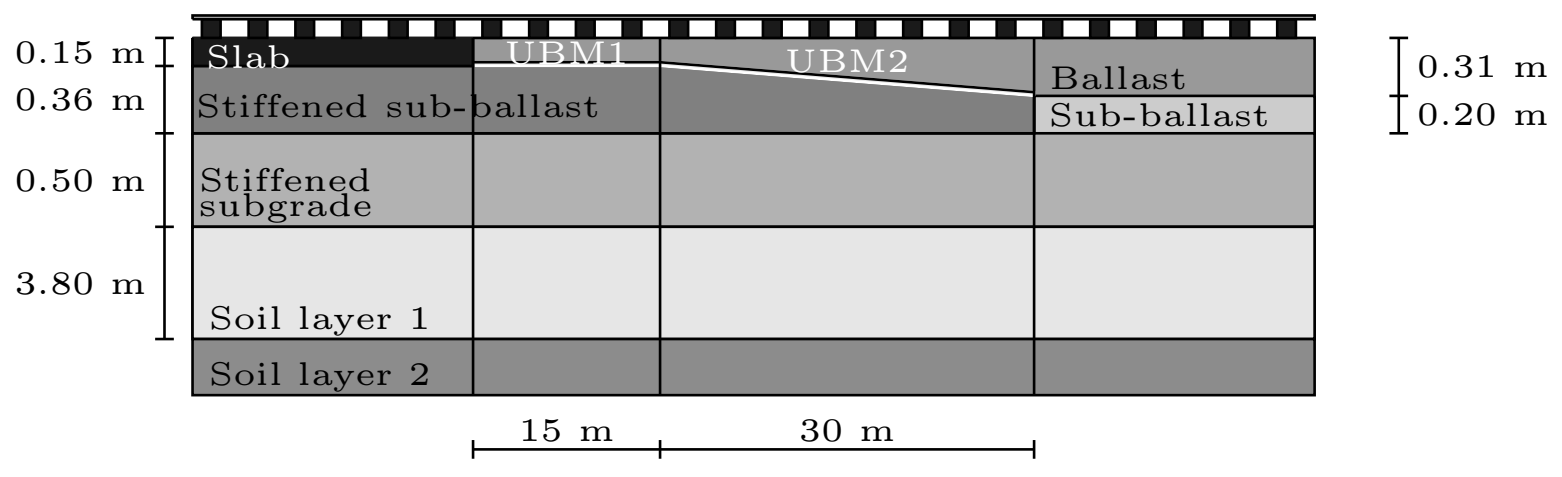

Figure 4: Longitudinal geometry of the transition zone in Chauconin [9].

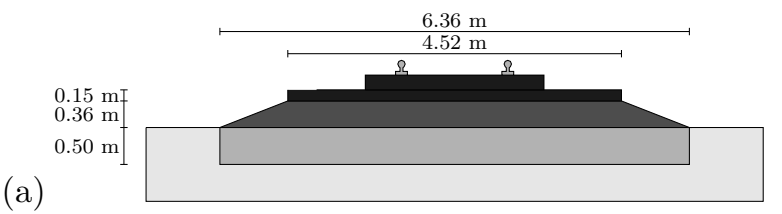

(b)

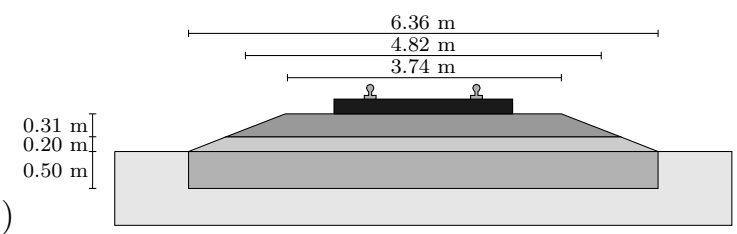

Figure 5: Cross sectional geometry of (a) the slab track and (b) the ballasted track [9].

A STEDEF [21] system (figure 5(a)) is used for the slab track. This system is very common in tunnels, particularly for metro systems, and on high speed train lines. The sleepers with spacing $L=0.60 \mathrm{~m}$ are supported by under sleeper pads enclosed in a rubber shoe, embedded in the concrete slab. The concrete sleeper blocks are enclosed between the rail pads and under sleeper pads, leading to a two-stage elasticity and, therefore, ensuring good noise and vibration insulation between the sleepers and the slab. The slab is supported by a layer of stiffened sub-ballast material. Underneath the ballast, the soil is stiffened up to a depth of $0.50 \mathrm{~m}$ over the entire width of the track (figure 5).

The ballasted track (figure 5(b)) consists of two UIC 60 rails connected to M450 monobloc sleepers by $9 \mathrm{~mm}$ thick Fastclip fastening systems. The sleepers are supported by a ballast layer on top of a layer of non-treated sub-ballast.

In the first part of the transition zone, the slab is replaced by a ballast layer. Between the ballast and the stiffened sub-ballast, a $2 \mathrm{~cm}$ thick under ballast mat (UBM) is placed to avoid a large stiffness variation at 
the beginning of the transition zone. The height of the ballast and sub-ballast layers is unchanged in this part. In the second part of the transition zone, a stiffer under ballast mat is used and the height of the ballast layer is gradually increased to smoothen the transition to the ballasted track. The properties of the materials used in the track are shown in tables 1 and 2 . The ballast track has softer rail pads than the slab track. The same sleepers are used in both track systems. The soil consists of a homogeneous layer on top of a halfspace.

\begin{tabular}{lccc}
\hline & & Slab track & Ballasted track \\
\hline Rail pad & $k_{\mathrm{rp}}$ & $150 \times 10^{6} \mathrm{~N} / \mathrm{m}$ & $60 \times 10^{6} \mathrm{~N} / \mathrm{m}$ \\
& $c_{\mathrm{rp}}$ & $120 \times 10^{3} \mathrm{Ns} / \mathrm{m}$ & $120 \times 10^{3} \mathrm{Ns} / \mathrm{m}$ \\
Sleeper & $b$ & $2.42 \mathrm{~m}$ & $2.42 \mathrm{~m}$ \\
& $l$ & $0.25 \mathrm{~m}$ & $0.25 \mathrm{~m}$ \\
& $m$ & $305 \mathrm{~kg}$ & $305 \mathrm{~kg}$ \\
Under sleeper pad & $k_{\text {usp }}$ & $32.5 \times 10^{6} \mathrm{~N} / \mathrm{m}$ & - \\
& $c_{\text {usp }}$ & $16.3 \times 10^{3} \mathrm{Ns} / \mathrm{m}$ & - \\
\hline
\end{tabular}

Table 1: Properties of the rail pads, sleepers and under sleeper pads.

\begin{tabular}{lcccc}
\hline Material & $\begin{array}{c}C_{\mathrm{s}} \\
{[\mathrm{m} / \mathrm{s}]}\end{array}$ & $\begin{array}{c}C_{\mathrm{p}} \\
{[\mathrm{m} / \mathrm{s}]}\end{array}$ & $\begin{array}{c}\beta_{\mathrm{s}}=\beta_{\mathrm{p}} \\
{[-]}\end{array}$ & $\begin{array}{c}\rho \\
{\left[\mathrm{kg} / \mathrm{m}^{3}\right]}\end{array}$ \\
\hline Ballast & 260.5 & 487.4 & 0.05 & 1700 \\
Sub-ballast & 176.7 & 367.8 & 0.02 & 2135 \\
Stiffened sub-ballast & 2144.8 & 3714.8 & 0.02 & 2000 \\
Slab & 2190.9 & 3794.7 & 0.02 & 2500 \\
Stiffened subgrade & 206.7 & 386.7 & 0.02 & 1800 \\
UBM 1 & 9.9 & 18.5 & 0.125 & 900 \\
UBM 2 & 11.9 & 22.2 & 0.125 & 900 \\
Soil layer 1 & 126.6 & 236.8 & 0.02 & 1800 \\
Soil layer 2 & 279.8 & 1998.3 & 0.02 & 1800 \\
\hline
\end{tabular}

Table 2: Dynamic material properties in the transition zone.

The track is split into 6 sections (figure 6): the slab track, the ballasted track and 4 sections corresponding 
to the transition between the two track systems. In reality, the sub-ballast height reduces gradually in the track section with UBM2 (figure 4). In the model, however, this track section is split into three parts to avoid that every cell should have a different geometry. In the first and third part, the height of the ballast and sub-ballast layers is constant, while the sub-ballast height decreases in the second part. This model simplification has only little effect on the results, as will be shown in section 3.2. Track sections 1 and 6 in figure 6 are semi-infinite, while sections 2 to 5 consist of 25, 24, 1 and 25 cells, respectively.

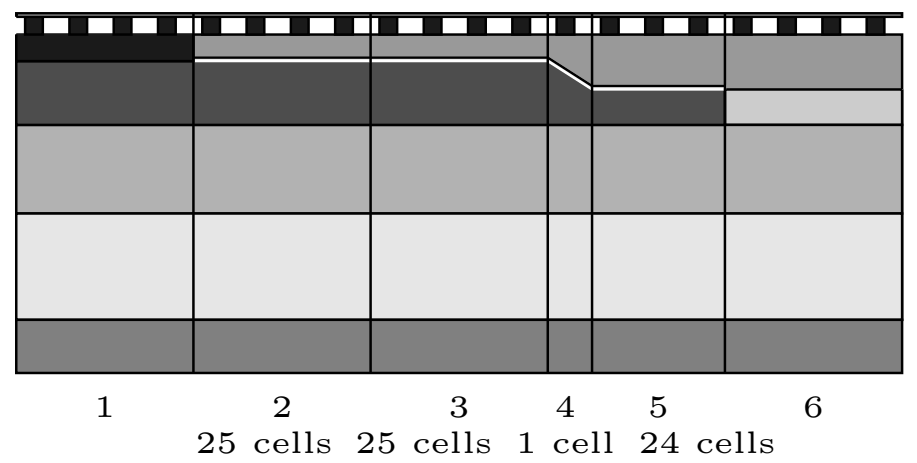

Figure 6: Division of the transition zone into 6 parts.

Each cell of the track is modeled as shown in figure 7 with quadratic 20-node finite elements. At the boundaries of the soil domain, a perfectly matched layer (PML) as implemented in [22] absorbs waves traveling in the $x$ - and $z$-direction. At least four elements per shear wave length are used for each frequency and for each material, both in the regular soil domain and inside the PML. The track model is symmetric around the $y z$-plane. To reduce the computation time, only half of the cell corresponding to positive $x$ coordinates is modeled and symmetric boundary conditions are imposed at $x=0$. Each cell has 2833 degrees of freedom, with 767 degrees of freedom at the interface between two cells.

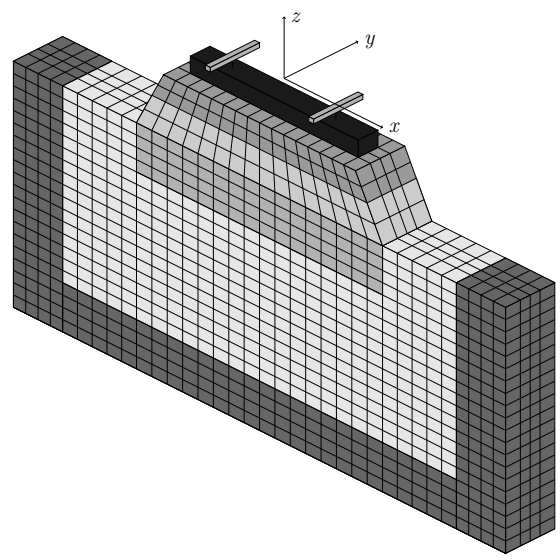

Figure 7: Model of one cell with perfectly matched layer. 
The track model based on the wave analysis approach can easily be combined with the two-step train-tracksoil coupling algorithm presented in [23]. In the first step of this algorithm, the train-track interaction is simulated and the interaction forces are computed in the time domain. In the second step, the track and free field vibrations are computed in the frequency domain by applying the computed train-track interaction forces to the rails of the track-soil model.

The interaction between the train and the track is simulated from $100 \mathrm{~m}$ before until $50 \mathrm{~m}$ after the transition zone with a time step $\Delta t=1 \times 10^{-4} \mathrm{~s}$. At the start of the calculation, the interaction forces are influenced by the sudden application of the train load on the track, leading to incorrect results in this part of the track. Therefore, the results in the first $50 \mathrm{~m}$ are disregarded. Computing the train-track interaction in these first $50 \mathrm{~m}$ could be avoided by slowly applying the train load on the track and making the train move only when train and track are in equilibrium. However, the computation time of the train-track coupling in the time domain is very short compared to the other steps in the coupling algorithm, so the relative reduction of the computation time would be small.

The displacements at the interface between the sub-ballast and the stiffened track subgrade are computed in the track section where the interaction force is applied on the track, as well as over a distance of $150 \mathrm{~m}$ at both sides of this track section. The regularity of the soil underneath this interface is exploited by transforming the interface displacements to the wavenumber domain and using a 2.5D FE-BE model of the stiffened subgrade and the soil to compute the corresponding tractions [24]. The free field vibrations are then computed from these interface displacements and tractions.

The transition zone is evaluated for the passage of a driving bogie of a TGV-Réseau train, operating on the LGV Est. The train is modeled as shown in figure 8 and consists of seven degrees of freedom: the vertical displacements of the car body, the bogie, two wheel sets and two wheel-rail contact points and the pitch rotation of the bogie. As the train and track are assumed to be symmetric and the track model consists of only one rail, the characteristics of the vehicle model correspond to one symmetric half of a bogie and one fourth of the car body. The car body and bogie in figure 8 have mass $m_{\mathrm{cb}}$ and $m_{\mathrm{b}}$, respectively, and are connected by the secondary suspension, modeled by a spring $k_{\mathrm{s} 2}$ and damper $c_{\mathrm{s} 2}$. The bogie with moment of inertia $I_{\mathrm{b}}$ is connected to the wheel sets with mass $m_{\mathrm{w}}$ by the primary suspension, modeled by a spring $k_{\mathrm{s} 1}$ and damper $c_{\mathrm{s} 1}$. The wheel sets are connected to massless wheel-rail contact points by linearised Hertzian springs $k_{\mathrm{H}}$. The characteristics of the different bogies are summarized in table 3 .

\subsection{Evaluation of the transition zone}

In this section, it is evaluated how the transition zone leads to a reduction of train-track interaction forces, as well as track and free field vibration. The effect of the transition zone on the train vibration is not taken into account in this paper. To evaluate the effect of the transition zone on passenger comfort, the proposed track model can be coupled to a more detailed train model, allowing for an accurate prediction of the car 


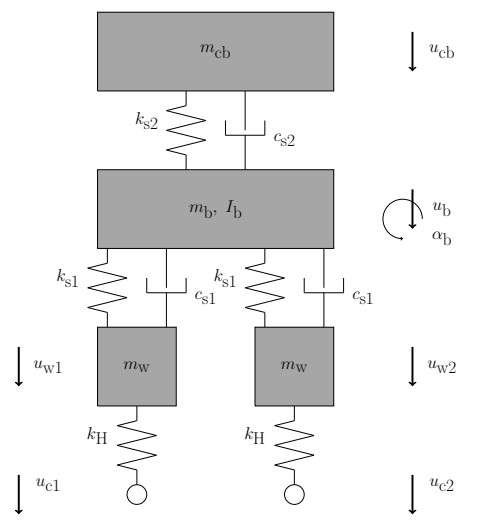

Figure 8: Vehicle model with seven degrees of freedom.

\begin{tabular}{lcc}
\hline Car body & $m_{\mathrm{cb}}$ & $13600 \mathrm{~kg}$ \\
Secondary suspension & $k_{\mathrm{s} 2}$ & $613 \times 10^{3} \mathrm{~N} / \mathrm{m}$ \\
& $c_{\mathrm{s} 2}$ & $10 \times 10^{3} \mathrm{Ns} / \mathrm{m}$ \\
Bogie & $m_{\mathrm{b}}$ & $1191 \mathrm{~kg}$ \\
& $I_{\mathrm{b}}$ & $750 \mathrm{kgm}^{2}$ \\
Primary suspension & $k_{\mathrm{s} 1}$ & $613 \times 10^{3} \mathrm{~N} / \mathrm{m}$ \\
& $c_{\mathrm{s} 1}$ & $5 \times 10^{3} \mathrm{Ns} / \mathrm{m}$ \\
Wheel set & $m_{\mathrm{ws}}$ & $512 \mathrm{~kg}$ \\
Hertzian spring & $k_{\mathrm{H}}$ & $1000 \times 10^{6} \mathrm{~N} / \mathrm{m}$ \\
Axle distance & $L_{\mathrm{a}}$ & $2.70 \mathrm{~m}$ \\
\hline
\end{tabular}

Table 3: Properties of the TGV Réseau vehicle model [25].

body acceleration.

The design of a transition zone is challenging due to the large difference in track stiffness between the two track systems. Figure 9 shows the variation of the static stiffness in the transition zone from slab track to ballasted track for a gradual change in track system, corresponding to the geometry in figure 6 , as well as for a sudden change in track system, where the slab track and the ballasted track are connected directly without any transition. It is observed that the static stiffness of the ballasted track is approximately twice as high as the slab track. The stiffening effect of the slab, giving a higher support stiffness to the sleepers in the slab track as compared to the ballast layer in the ballasted track, is compensated by the soft under sleeper pads, making the slab track softer than the ballasted track.

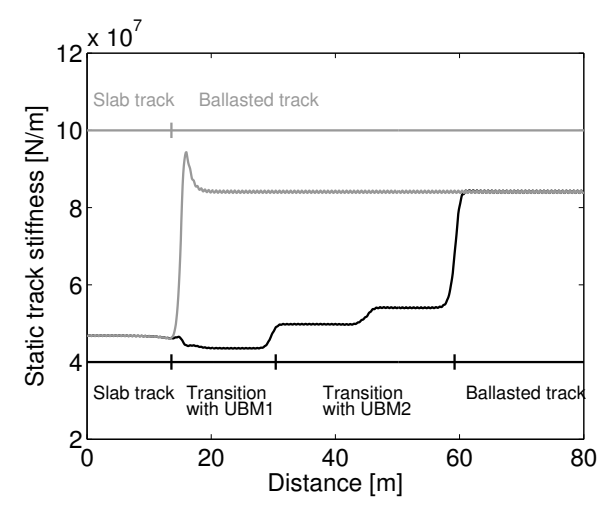

Figure 9: Static track stiffness in the transition zone in case of a gradual (black line) and sudden (grey line) change in track system. 
In case of a sudden change in track system (grey line in figure 9), the track stiffness changes with $50 \mathrm{MN} / \mathrm{m}$ at the entry of the ballasted track zone. The track stiffness increases to a value which is higher than the actual stiffness of the ballasted track. The sleeper support stiffness in this part of the ballasted track is influenced by the close proximity of the slab, leading to a stiffer support for the sleepers than a continuous ballast layer further away from the transition.

In case of a gradual transition between the two track systems (black line in figure 9), the track stiffness changes in four steps. In the first step, the track stiffness is slightly reduced as a result of the transition from the slab track to the ballasted track with UBM1. The second step is due to the change from the part with UBM1 to the part with the stiffer UBM2. The third step results from approximating the gradual reduction of the ballast layer thickness as shown in figure 6. In the fourth step, the transition from the part with UBMs to a part without UBM leads to the largest stiffness change. The stiffness variation at the end of the slab track is highly reduced in this case, since the effect of the soft under sleeper pads is compensated by the low sleeper support stiffness in the part of the track with UBM1. This transition zone therefore leads to a much more gradual change in track stiffness.

Figure 10 shows the vertical rail receptance above a sleeper for slab track and ballasted track. The receptance of the slab track is clearly much higher in the whole frequency range between 0 and $100 \mathrm{~Hz}$. The peak around $50 \mathrm{~Hz}$ corresponds to the resonance frequency of the rails and sleepers on the under sleeper pads. For the ballasted track, the small peak around $10 \mathrm{~Hz}$ is due to the soil stratification. This peak is also present in the receptance of the slab track, but is barely observed. The P2 resonance frequency of the vehicle on the slab track and on the ballasted track is around $30 \mathrm{~Hz}$ and $55 \mathrm{~Hz}$, respectively. The $\mathrm{P} 2$ resonance frequency on the slab track is relatively low as a result of the high track receptance due to the soft under sleeper pads.

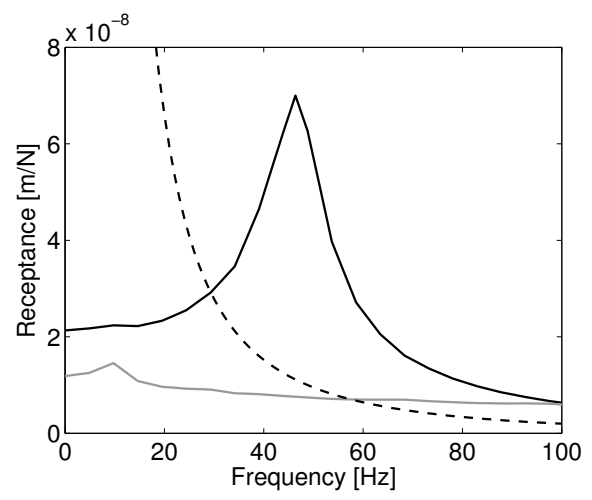

Figure 10: Vertical rail receptance above a sleeper of the slab track (solid black line) and the ballasted track (solid grey line) and receptance of the vehicle (dashed black line).

The passage of a driving bogie of a TGV-Réseau train traveling at $300 \mathrm{~km} / \mathrm{h}$ is simulated. This is done for 
the case with the gradual change in track system as well as for a sudden transition between the two track systems. The influence of the running direction (slab-ballast and ballast-slab) is also investigated.

Figure 11 shows the time history and frequency spectrum of the dynamic train-track interaction forces for the transition from slab track to ballasted track. In case of a sudden stiffness variation, a dynamic peak force up to $8000 \mathrm{~N}$ is observed at the transition between the two track systems. In the frequency spectrum, all frequencies up to $150 \mathrm{~Hz}$ are excited with a peak around $35 \mathrm{~Hz}$, close to the P2 resonance frequency of the vehicle on the slab track. The peak around $140 \mathrm{~Hz}$ corresponds to the sleeper passing frequency. In case of a gradual change in track stiffness, peaks in the dynamic interaction force are observed at three positions: at the end of the slab track, at the transition from the track with UBM1 to the track with UBM2 and at the beginning of the ballasted track. The peak forces are clearly reduced due to the gradual transition between the track systems. A small dynamic excitation is observed in the middle of the track section with UBM2, where the height of the sub-ballast layer is reduced in one cell (figure 6). The effect of this model simplification is found to be small.

(a)

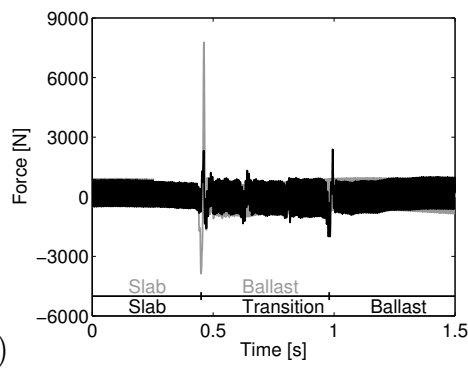

(b)

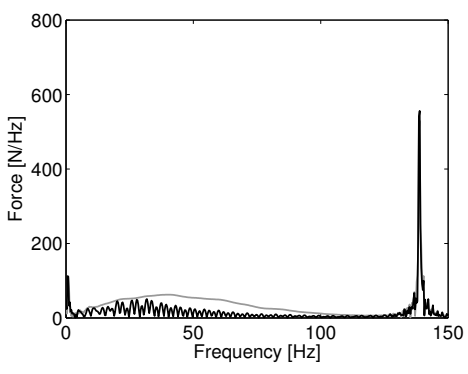

Figure 11: (a) Time history and (b) frequency spectrum of the dynamic train-track interaction forces for a train traveling at $300 \mathrm{~km} / \mathrm{h}$ from slab track to ballasted track in case of a gradual (black line) and sudden (grey line) transition.

Figure 12 shows the time history and frequency spectrum of the dynamic train-track interaction forces for a train traveling in the other direction, from ballasted track to slab track. For a sudden stiffness change, a dynamic peak force around $5000 \mathrm{~N}$ is observed. This is lower than the peak force for a train going from slab track to ballasted track, but it takes longer for the interaction force to return to its steady state. In the frequency domain, a peak is observed around $35 \mathrm{~Hz}$. In case of a gradual change in track system, the peak forces are clearly reduced.

Figure 13 shows spectrograms with a time window of $0.02 \mathrm{~s}$ of the dynamic train-track interaction forces for a train traveling from slab track to ballasted track at $300 \mathrm{~km} / \mathrm{h}$, for a gradual as well as a sudden change in track system. The color scale is the same for both figures. Figure 13 clearly illustrates the locations of the highest interaction forces. In case of a sudden track stiffness variation, very high interaction forces are observed in a very broad frequency band. In case of a gradual change, high interaction forces are observed 


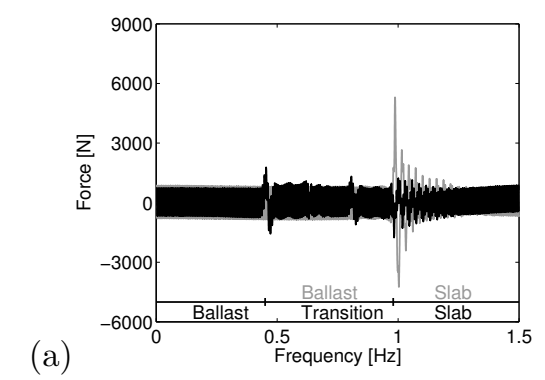

(b)

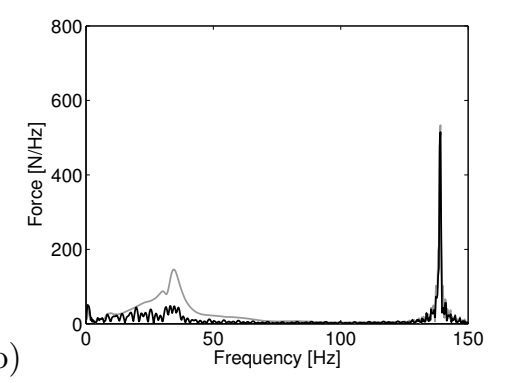

Figure 12: (a) Time history and (b) frequency spectrum of the dynamic train-track interaction forces for a train traveling at $300 \mathrm{~km} / \mathrm{h}$ from ballasted track to slab track in case of a gradual (black line) and sudden (grey line) transition.

at three locations, but their peak values are lower and they are concentrated in a more limited frequency band up to $100 \mathrm{~Hz}$.

(a)
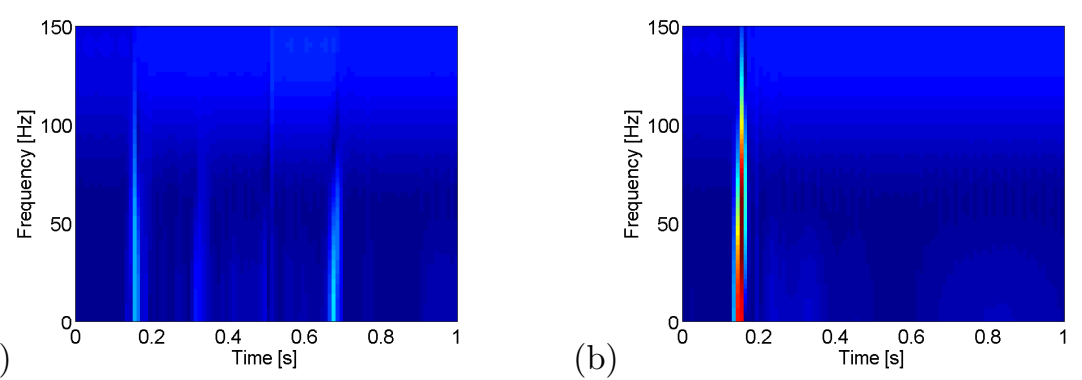

Figure 13: Spectrogram of the dynamic train-track interaction forces for a train traveling at $300 \mathrm{~km} / \mathrm{h}$ from slab track to ballasted track in case of a (a) gradual and (b) sudden transition.

Figure 14 shows spectrograms with a time window of $0.02 \mathrm{~s}$ of the dynamic train-track interaction forces for a train traveling from ballasted track to slab track at $300 \mathrm{~km} / \mathrm{h}$, for a gradual as well as a sudden change in track system. The color scale is the same for both figures and the same as in figure 13 . For a sudden transition, high interaction forces are observed. Compared to the transition from slab track to ballasted track (figure 13), the peak value of the interaction force is lower and the highest dynamic forces are concentrated below $100 \mathrm{~Hz}$. The time period with high interaction forces is longer, however.

At the transition zone in Chauconin, rapid track degradation was observed due to the passage of high speed trains at $300 \mathrm{~km} / \mathrm{h}$. Therefore, the maximum train speed was reduced. Figure 15 shows the time history and frequency spectrum of the dynamic train-track interaction forces for a train traveling at $200 \mathrm{~km} / \mathrm{h}$ on the transition from slab track to ballasted track. Compared to a train passing at $300 \mathrm{~km} / \mathrm{h}$, the peak forces are reduced by more than half, both for a sudden and gradual change in track system. The peak in the frequency spectrum around $95 \mathrm{~Hz}$ corresponds to the sleeper passing frequency. 


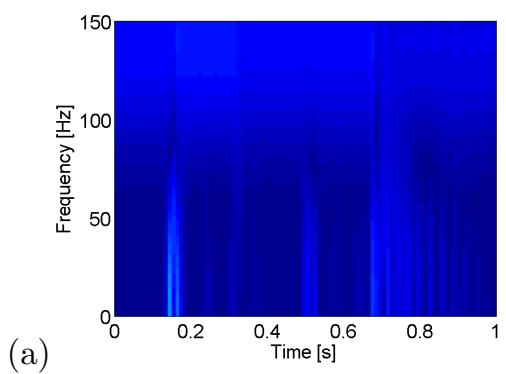

(b)

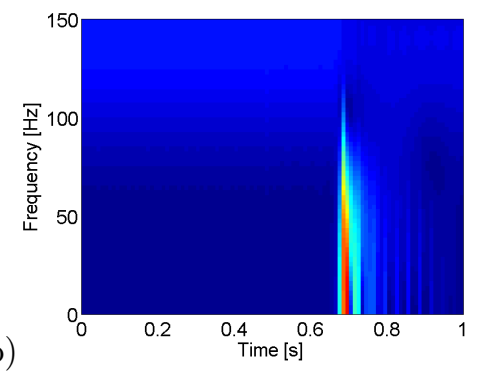

Figure 14: Spectrogram of the dynamic train-track interaction forces for a train traveling at $300 \mathrm{~km} / \mathrm{h}$ from ballasted track to slab track in case of a (a) gradual and (b) sudden transition.
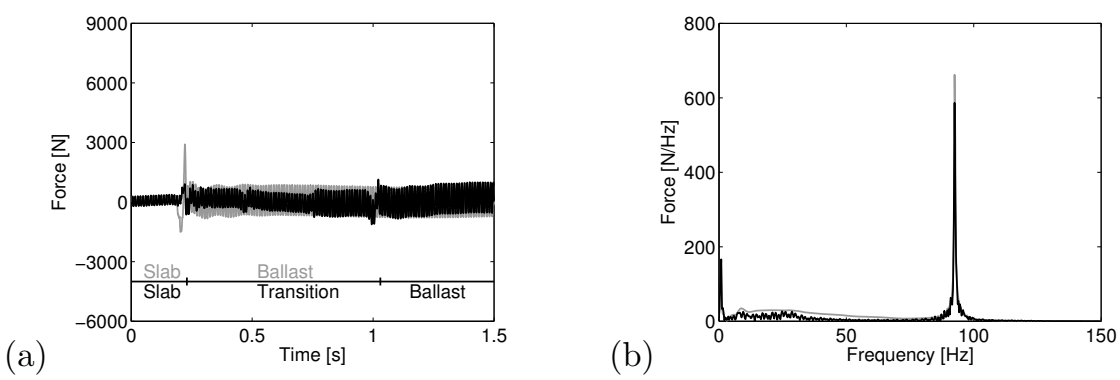

Figure 15: (a) Time history and (b) frequency spectrum of the dynamic train-track interaction forces for a train traveling at $200 \mathrm{~km} / \mathrm{h}$ from slab track to ballasted track in case of a gradual (black line) and sudden (grey line) transition.

Figure 16 shows the time history and frequency spectrum of the dynamic train-track interaction forces for a train traveling at $200 \mathrm{~km} / \mathrm{h}$ on the transition from ballasted track to slab track. The peak forces are here reduced as well, when compared to those for a train traveling at $300 \mathrm{~km} / \mathrm{h}$. It is observed that at $200 \mathrm{~km} / \mathrm{h}$ the peak force has almost the same value for trains running in both directions.

Figure 17 shows the maximum vibration velocity of the sleeper and the track directly under the sleeper during the passage of the train at $300 \mathrm{~km} / \mathrm{h}$ from slab track to ballasted track. A difference between the vibration velocity of the sleepers and the track directly underneath them is only observed for the slab track, due to the presence of the under sleeper pads. Figure 17 clearly shows that the highest sleeper velocities are observed at the end of the slab track, both for a gradual and a sudden change in track system. The under sleeper pads lead to a dynamic decoupling between the sleepers and the concrete slab. For a sudden change in track system, higher sleeper vibrations are observed in both slab track and ballasted track.

Figure 18(a) shows the time history of the vertical vibration velocity of the most heavily excited sleeper in the slab track. For a gradual change in track stiffness, the quasi-static effect of each of the two passing axles can clearly be observed. The dynamic excitation is superimposed on this quasi-static vibration, but is limited in this case. For a sudden change in track system, a large dynamic excitation is observed when 


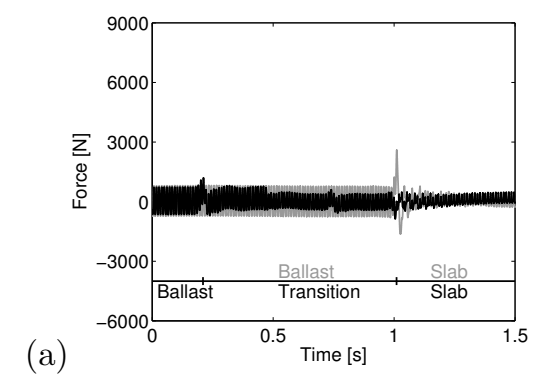

(b)

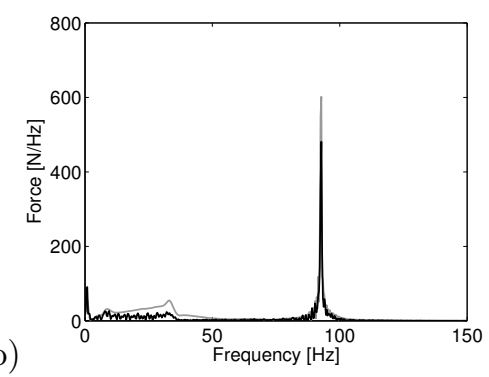

Figure 16: (a) Time history and (b) frequency spectrum of the dynamic train-track interaction forces for a train traveling at $200 \mathrm{~km} / \mathrm{h}$ from ballasted track to slab track in case of a gradual (black line) and sudden (grey line) transition.

(a)

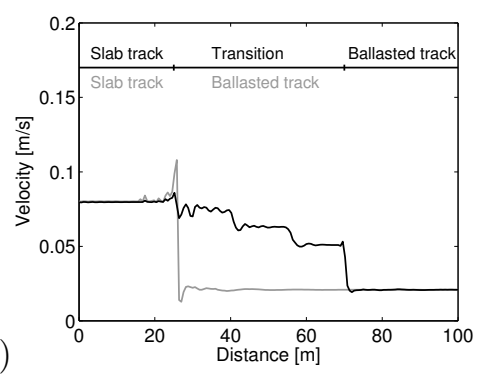

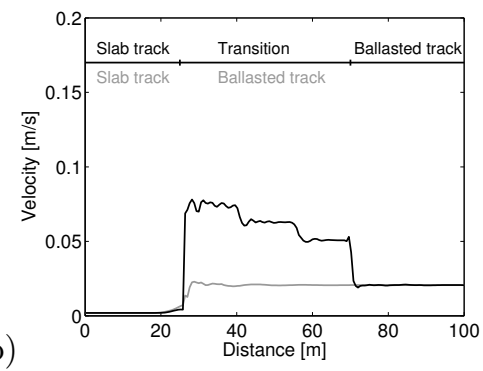

Figure 17: Maximum vibration velocity of (a) the sleepers and (b) the track directly underneath the sleepers during the passage of the train at $300 \mathrm{~km} / \mathrm{h}$ from slab track to ballasted track in case of a gradual (black line) and sudden (grey line) change in track system.

the first axle moves from the slab track to the ballasted track, around $0.45 \mathrm{~s}$. Figure 18(b) shows the time history of the vertical vibration velocity of the track directly underneath this sleeper. The vibration velocity of the concrete slab is much lower than the vibration velocity of the sleepers due to the presence of the under sleeper pads. These pads also distribute the train load over a larger section of the track; therefore, the individual passages of the two axles are not observed in the vibration velocity of the slab. A sudden change in track stiffness results in significantly higher dynamic excitation of the slab, especially between $0.45 \mathrm{~s}$ and $0.55 \mathrm{~s}$.

Figure 19 shows the time history of the vertical vibration velocity of the most heavily excited sleeper in the ballasted track. The passage of the individual axles is clearly observed. Due to the much stiffer sleeper support in the ballasted track, the sleeper vibration is much lower than in the slab track. The quasi-static excitation dominates the sleeper response. The dynamic excitation results in higher vibrations in case of a sudden change in track system, but the contribution to the total sleeper velocity is relatively small.

Figures 20 and 21 show the time history and frequency spectrum of the free field vibration velocity at $6 \mathrm{~m}$ and $12 \mathrm{~m}$ from the track center line, at the end of the slab track. The quasi-static effect of the passing 


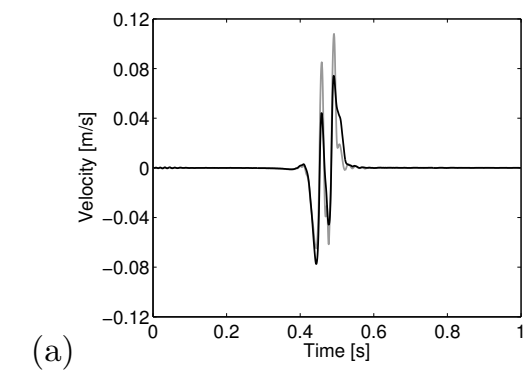

(b)

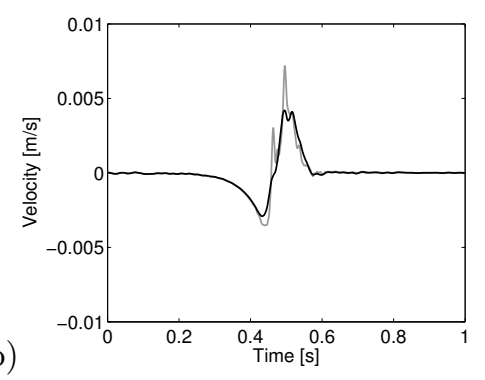

Figure 18: Time history of the vertical vibration velocity of (a) the most excited sleeper in the slab track and (b) the track directly under this sleeper during the passage of the train at $300 \mathrm{~km} / \mathrm{h}$ from slab track to ballasted track in case of a gradual (black line) and sudden (grey line) change in track system.

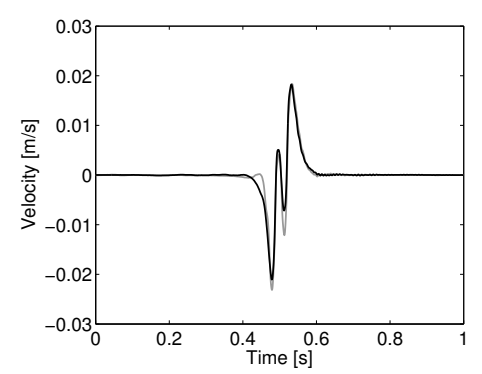

Figure 19: Time history of the vertical vibration velocity of the most excited sleeper in the ballasted track during the passage of the train at $300 \mathrm{~km} / \mathrm{h}$ from slab track to ballasted track in case of a gradual (black line) and sudden (grey line) change in track system.

train is clearly visible and decreases with increasing distance from the track. The passage of the individual axles is not observed. The dynamic excitation is larger in case of a sudden change in track system, but the difference is relatively small. In the frequency spectrum, a difference between the free field response for a sudden and gradual change in track system is observed above $60 \mathrm{~Hz}$.

The transition zone in Chauconin results in a much smoother transition between the slab track and the ballasted track. The train-track interaction forces and the vibrations of the sleepers and the track are clearly reduced.

\section{Conclusions}

A track modeling approach based on a wave analysis technique for multi-coupled periodic structures is presented. A track with varying characteristics in the longitudinal direction can be modeled to predict train-track interaction forces and track and free field vibrations due to parametric excitation. The track is divided into parts with different properties, each consisting of a number of periodic cells. The response of 
(a)

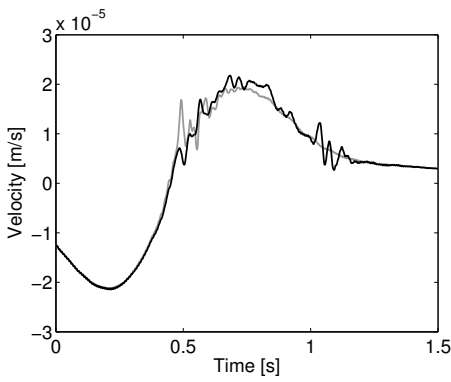

(b)

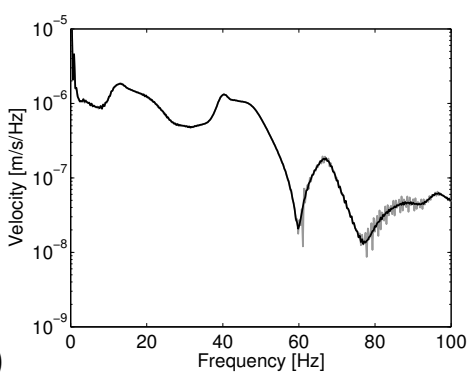

Figure 20: (a) Time history and (b) frequency spectrum of the free field velocity at $6 \mathrm{~m}$ from the track center line during the passage of the train at $300 \mathrm{~km} / \mathrm{h}$ from slab track to ballasted track in case of a gradual (black line) and sudden (grey line) change in track system.

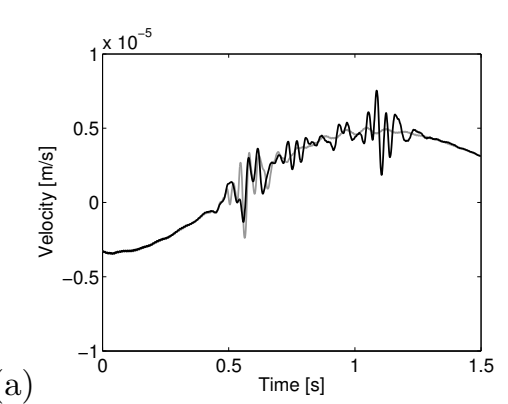

(a) (b)

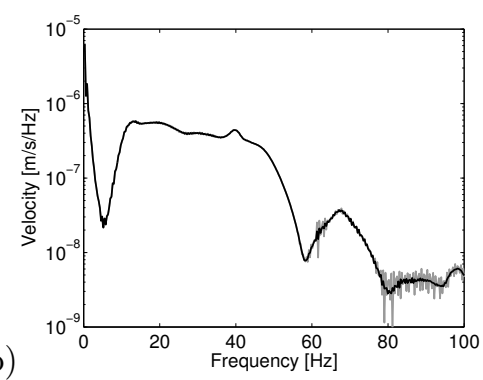

Figure 21: (a) Time history and (b) frequency spectrum of the free field velocity at $12 \mathrm{~m}$ from the track center line during the passage of the train at $300 \mathrm{~km} / \mathrm{h}$ from slab track to ballasted track in case of a gradual (black line) and sudden (grey line) change in track system.

the track to a force applied on the rail is computed by studying the free wave propagation in all cells left and right of the excited cell. It is demonstrated how these free waves are computed and how they are used to assemble a system of equations that yields the displacements in the entire track structure. When forces are applied on different cells, the computation time can be drastically reduced by maximally recuperating the computations made for previous cells.

The capability of the model to predict train-track interaction forces, track vibration and free field vibration due to parametric excitation is demonstrated in a case study. The performance of a transition zone between a slab track and a ballasted track in Chauconin, France, is evaluated during the passage of a high speed passenger train. The results are compared to a case where no specific measures are taken between the slab track and the ballasted track. The highest peak forces are observed for a train traveling from slab track to ballasted track. The train-track interaction forces and the vibration of the sleepers, of the concrete slab and in the free field are clearly reduced as a result of the carefully designed transition zone. 


\section{Acknowledgements}

The first author is a PhD student supported by the Research Foundation Flanders (FWO). The financial support is gratefully acknowledged.

\section{References}

[1] B. Coelho, Dynamics of railway transition zones in soft soils, Ph.D. thesis, TU Delft, 2011.

[2] T. Ekevid, N. Wiberg, Wave propagation related to high-speed train. A scaled boundary FE-approach for unbounded domains, Computer Methods in Applied Mechanics and Engineering 191 (2002) 3947-3964.

[3] P. Galvín, A. Romero, J. Domínguez, Fully three-dimensional analysis of high-speed train-track-soil-structure dynamic interaction, Journal of Sound and Vibration 329 (2010) 5147-5163.

[4] J. O'Brien, D. Rizos, A 3D FEM-BEM methodology for simulation of high speed train induced vibrations, Soil Dynamics and Earthquake Engineering 25 (2005) 289-301.

[5] D. Aubry, D. Clouteau, G. Bonnet, Modelling of wave propagation due to fixed or mobile dynamic sources, in: N. Chouw, G. Schmid (Eds.), Workshop Wave '94, Wave propagation and Reduction of Vibrations, Ruhr Universität Bochum, Germany, 1994, pp. 109-121.

[6] D. Clouteau, M. Arnst, T. Al-Hussaini, G. Degrande, Freefield vibrations due to dynamic loading on a tunnel embedded in a stratified medium, Journal of Sound and Vibration 283 (2005) 173-199.

[7] D. Clouteau, M. Elhabre, D. Aubry, Periodic BEM and FEM-BEM coupling: application to seismic behaviour of very long structures, Computational Mechanics 25 (2000) 567-577.

[8] G. Degrande, D. Clouteau, R. Othman, M. Arnst, H. Chebli, R. Klein, P. Chatterjee, B. Janssens, A numerical model for ground-borne vibrations from underground railway traffic based on a periodic finite element - boundary element formulation, Journal of Sound and Vibration 293 (2006) 645-666.

[9] E. Arlaud, Modèles dynamiques réduits de milieux périodiques par morceaux : application aux voies ferroviaires, Ph.D. thesis, l'École Nationale Supérieure d'Arts et Métiers, 2016.

[10] E. Arlaud, S. Costa D'Aguar, E. Balmes, G. Faussurier, Numerical study of railway track dynamics: Case of a transition zone, Engineering Analysis with Boundary Elements 27 (2016) 23-38.

[11] G. Kouroussis, D. Connolly, G. Alexandrou, K. Vogiatzis, Railway ground vibrations induced by wheel and rail singular defects, Vehicle System Dynamics 53 (2015) 1500-1519.

[12] D. Mead, The forced vibration of one-dimensional multi-coupled periodic structures: An application to finite element analysis, Journal of Sound and Vibration 319 (2009) 282-304.

[13] M. Germonpré, G. Degrande, G. Lombaert, A track model for railway-induced ground vibration resulting from a transition zone, Journal of Rail and Rapid Transit 232 (2018) 1703-1717.

[14] J. Fan, C. Zhou, J. Laine, M. Ichchou, L. Li, Model reduction schemes for the wave and finite element method using the free modes of a unit cell, Computers and Structures 197 (2018) 42-57.

[15] C. Esveld, Modern railway track. Second Edition., MRT-Productions, Zaltbommel, 2001.

[16] M. Steenbergen, A. Metrikine, C. Esveld, Assessment of design parameters of a slab track railway system from a dynamic viewpoint, Journal of Sound and Vibration 306 (2007) 361-371.

[17] M. Shahraki, C. Warnakulasooriya, K. Witt, Numerical study of transition zone between ballasted and ballastless railway track, Transportation Geotechnics 3 (2015) 58-67.

[18] A. Paixão, E. Fortunato, R. Calçada, The effect of differential settlements on the dynamic response of the train-track system: A numerical study, Engineering Structures 88 (2015) 216-224. 
[19] Y. Momoya, T. Takahashi, T. Nakamura, A study on the deformation characteristics of ballasted track at structural transition zone by multi-actuator moving loading test apparatus, Transportation Geotechnics 6 (2016) 123-134.

[20] R. Sañudo, L. dell'Olio, J. Casado, I. Carrascal, S. Diego, Track transitions in railways: A review, Construction and Building Materials 112 (2016) 140-157.

[21] L. Dieleman, M. Fumey, A. Robinet, P. Ramondec, D. Martin, Experimentation of a track section without ballast on the newline of East European TGV, in: Proceedings of WCRR2008 - World Congress on Railway Research, 2008.

[22] M. Papadopoulos, S. François, G. Degrande, G. Lombaert, The influence of uncertain local subsoil conditions on the response of buildings to ground vibration, Journal of Sound and Vibration 418 (2018) 200-220.

[23] M. Germonpré, G. Degrande, G. Lombaert, A study of modelling simplifications in ground vibration predictions for railway traffic at grade, Journal of Sound and Vibration 406 (2017) 208-223.

[24] S. François, M. Schevenels, G. Lombaert, P. Galvín, G. Degrande, A 2.5D coupled FE-BE methodology for the dynamic interaction between longitudinally invariant structures and a layered halfspace, Computer Methods in Applied Mechanics and Engineering 199 (2010) 1536-1548.

[25] P. Ferreira, Modelling and Prediction of the Dynamic Behaviour of Railway Infrastructures at Very High Speeds, Ph.D. thesis, Universidade de Lisboa, 2010. 